\title{
Benchmarking the Pure Random Search on the BBOB-2009 Testbed
}

\author{
Anne Auger \\ TAO Team - INRIA Saclay \\ LRI, Bat 490, Univ. Paris-Sud \\ 91405 Orsay Cedex, France \\ anne.auger@inria.fr
}

\author{
Raymond Ros \\ Univ. Paris-Sud, LRI \\ UMR 8623 / INRIA Saclay, projet TAO \\ F-91405 Orsay, France \\ raymond.ros@Iri.fr
}

\begin{abstract}
We benchmark the pure random search algorithm on the BBOB 2009 noise-free testbed. Each candidate solution is sampled uniformly in $[-5,5]^{D}$, where $D$ denotes the search space dimension. The maximum number of function evaluations chosen is $10^{6}$ times the search space dimension. With this budget the algorithm is not able to solve any single function of the testbed.

\section{Categories and Subject Descriptors}

G.1.6 [Numerical Analysis]: OptimizationGlobal Optimization, Unconstrained Optimization; F.2.1 [Analysis of Algorithms and Problem Complexity]: Numerical Algorithms and Problems
\end{abstract}

\section{General Terms}

Algorithms

\section{Keywords}

Benchmarking, Pure random search, Monte-Carlo, Blackbox optimization, Evolutionary computation

\section{INTRODUCTION}

The pure random search, first proposed by Brooks in 1958 [1], is the most simple stochastic search algorithm that consists in sampling each search point independently in the search domain and keeping the best solution found.

\section{METHODS}

We have used a uniform sampling in $[-5,5]^{D}$, where $D$ denotes the dimension of the search space. The experiments according to [3] on the benchmark functions given in $[2,4]$ have been conducted using both a C-code and a Matlab code. The algorithm implementation in Matlab is given in Figure 1. A maximum of $10^{6} \times D$ function evaluations has

Permission to make digital or hard copies of all or part of this work for personal or classroom use is granted without fee provided that copies are not made or distributed for profit or commercial advantage and that copies bear this notice and the full citation on the first page. To copy otherwise, to republish, to post on servers or to redistribute to lists, requires prior specific permission and/or a fee.

GECCO'09, July 8-12, 2009, Montréal Québec, Canada.

Copyright 2009 ACM 978-1-60558-505-5/09/07 ...\$5.00.
Figure 1: Pure random search in Matlab. At each iteration (iter), 200 points are sampled and stored in a matrix of size $D \times 200$ so as to reduce loops and function calls within Matlab.

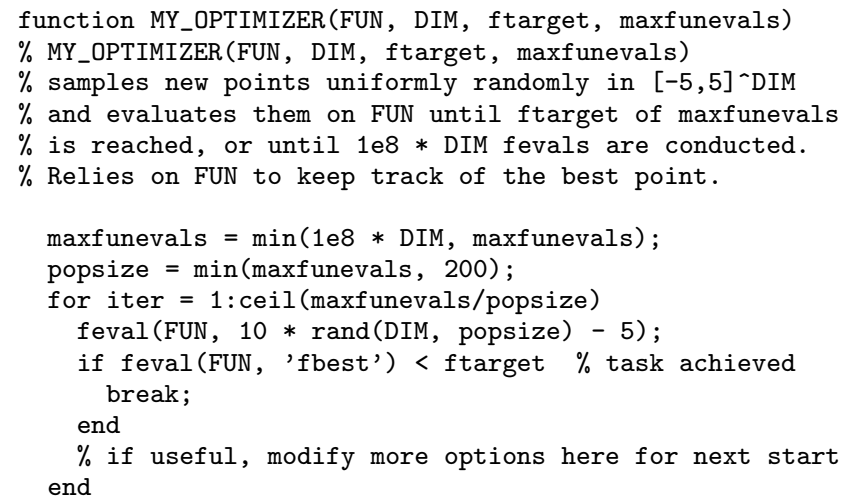

been used. The Matlab code was used for previous experiments using $10^{5} \times D$ function evaluations. The simulations for $2 ; 5 ; 10$ and $20 \mathrm{D}$ with $10^{5} \times D$ function evaluations with the C-code took 21 hours. The $40 \mathrm{D}$ experiments with $10^{5} \times D$ function evaluations in Matlab took 17 hours. Experiments with $10^{5} \times D$ function evaluations were done on the machine described in Section 4. The experiments with $10^{6} \times D$ function evaluations were done with the C-code on a Intel Xeon 2.00GHz: the experiments till dimension 20 took 38 hours and the 40-D experiments took 116 hours.

No parameter tuning was done and the crafting effort $\mathrm{CrE}$ [3] is computed to zero.

\section{RESULTS AND DISCUSSION}

Results from experiments according to [3] on the benchmark functions given in $[2,4]$ are presented in Figures 3 and 4 and in Table 1.

We see that with $10^{6} \times D$ function evaluations, the pure random search algorithm is not able to solve any function. However, since we use a uniform sampling in the search domain, we obtain as a by-product of the results an estimate of the volume of the sublevel sets: the sublevel sets of a function $f: \mathbb{R}^{D} \rightarrow \mathbb{R}$ are defined as $S_{c}=\left\{x \in \mathbb{R}^{D} \mid f(x) \leq c\right\}$ for $c$ spanning $\mathbb{R}$. If $S_{c}$ is a subset of $[-5,5]^{D}$, the hitting 


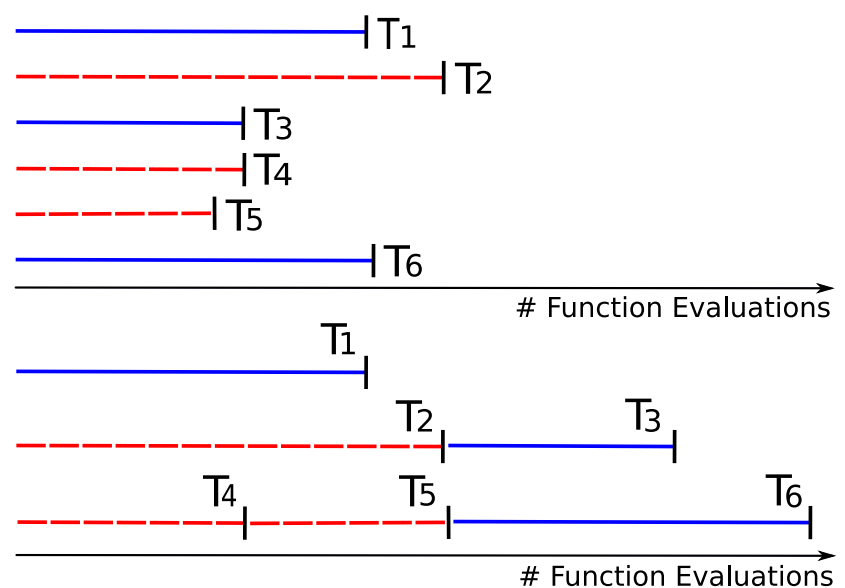

Figure 2: Illustration that $E R T(\Delta f)$ estimates the expected hitting time of an algorithm restarted until success (assuming infinite horizon): among 6 runs of the same algorithm $A$, the 1st, 3rd and 6th are successful while the 2nd, 4 th and 5 th are unsuccessful and thus $T_{1}, T_{2}+T_{3}$ and $T_{4}+T_{5}+T_{6}$ are 3 instances of the algorithm restart-A (i.e., algorithm $A$ restarted until success). Thus an estimate of the expected hitting time of restart- $\mathbf{A}$ is $\left(T_{1}+\left(T_{2}+T_{3}\right)+\left(T_{4}+T_{5}+T_{6}\right)\right) / 3$, i.e., total number of function evaluations divided by number of successes of algorithm A, i.e., $E R T(\Delta f)$. In the case where algorithm $A$ is the pure random search, the picture is simpler because unsuccessful runs always reach the maximum number of evaluations and thus the $2 \mathrm{nd}, 4 \mathrm{th}$ and 5 th runs have the same length. $T_{1}, T_{2}+T_{3}$ and $T_{4}+T_{5}+T_{6}$ represent then 3 instances of the pure random search that would be run with infinite horizon until a success is reached and $E R T(\Delta f)$ estimates thus the expected hitting time of the pure random search with infinite horizon.

time $T_{c}$ (assuming infinite horizon) of the sublevel set $S_{c}$ is distributed according to a geometric random variable of parameter $p_{c}=\operatorname{Vol}\left(S_{c}\right) / \operatorname{Vol}\left([-5,5]^{D}\right)$. The expected running time $\operatorname{ERT}(\Delta f)$ estimates the expected value of $T_{\Delta f}$ (see Figure 2), that equals $1 / p_{c}$ since $T_{\Delta f}$ is a geometric random variable. And thus $\operatorname{ERT}(\Delta f)$ gives the ratio between $\operatorname{Vol}\left([-5,5]^{D}\right)$ and $\operatorname{Vol}\left(S_{c}\right)$.

\section{CPU TIMING EXPERIMENT}

For the timing experiment the pure random search was run with a maximum of $10^{5} \times D$ function evaluations and restarted until 30 seconds has passed (according to Figure 2 in [3]). The experiments have been conducted with an Intel Core 2 Duo 2.53 GHz under Mac OS X Version 10.5.6 using the C-code provided. The time per function evaluation was $2.0 ; 2.3 ; 2.8 ; 4.2 ; 6.9$ times $10^{-7}$ seconds in dimensions $2 ; 3$; $5 ; 10 ; 20 ; 40$ respectively.

\section{CONCLUSION}

We have presented the results of the pure random search, a non-adaptive algorithm, that does not use information gathered during search for guiding its next steps. The performance is poor and expected to be outperformed by any reasonable algorithm. Furthermore, those results constitute reference results useful for the investigation of more advanced algorithms.

\section{Acknowledgments}

We would like to thank Nikolaus Hansen for the way he led the BBOB project, Steffen Finck and Nikolaus Hansen for their great and hard work. We also would like to thank Marc Schoenauer for his kind support and essential help on the $\mathrm{C}$-code.

\section{REFERENCES}

[1] S. H. Brooks. A discussion of random methods for seeking maxima. Operations Research, 6:244- 251, 1958.

[2] S. Finck, N. Hansen, R. Ros, and A. Auger. Real-parameter black-box optimization benchmarking 2009: Presentation of the noiseless functions. Technical Report 2009/20, Research Center PPE, 2009.

[3] N. Hansen, A. Auger, S. Finck, and R. Ros. Real-parameter black-box optimization benchmarking 2009: Experimental setup. Technical Report RR-6828, INRIA, 2009.

[4] N. Hansen, S. Finck, R. Ros, and A. Auger. Real-parameter black-box optimization benchmarking 2009: Noiseless functions definitions. Technical Report RR-6829, INRIA, 2009. 

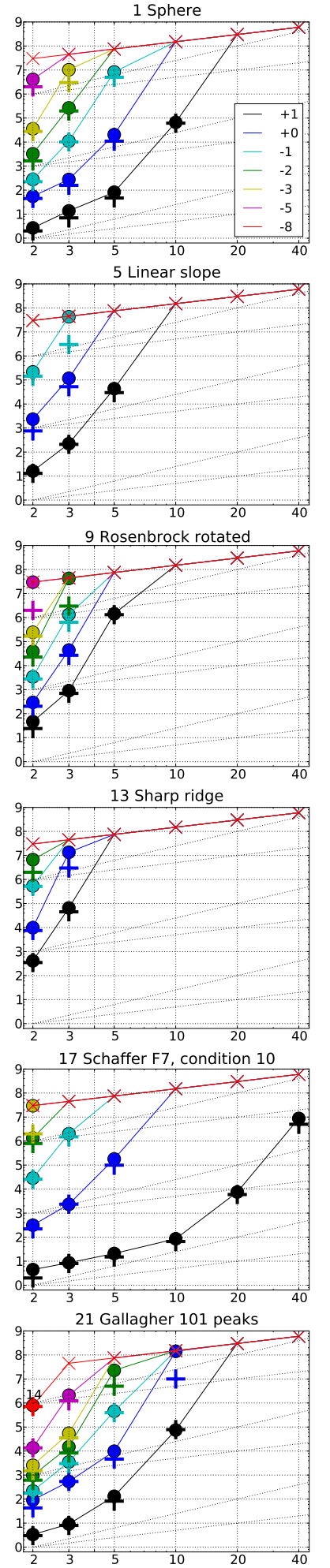
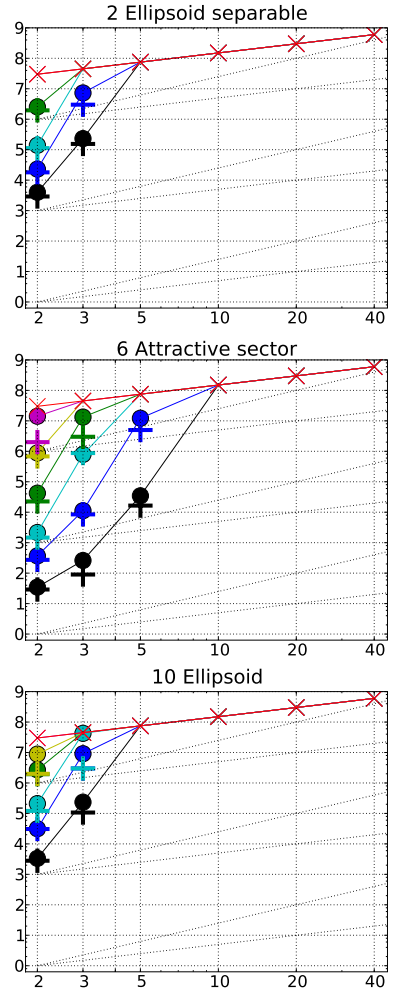

14 Sum of different powers
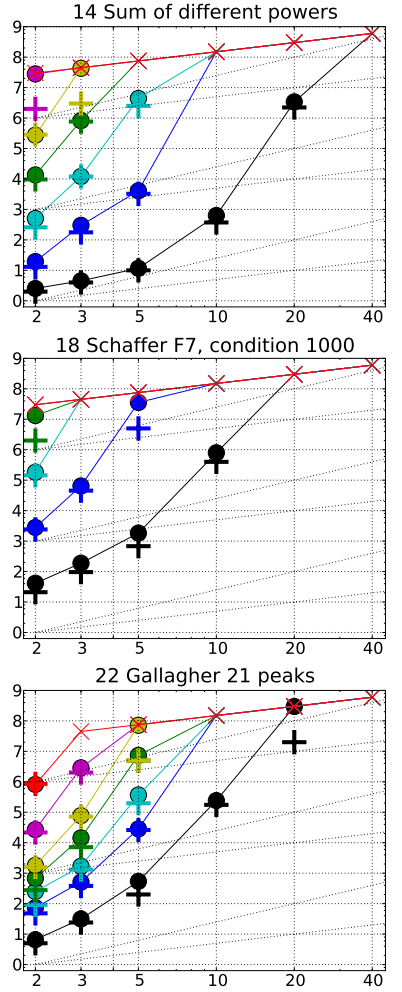
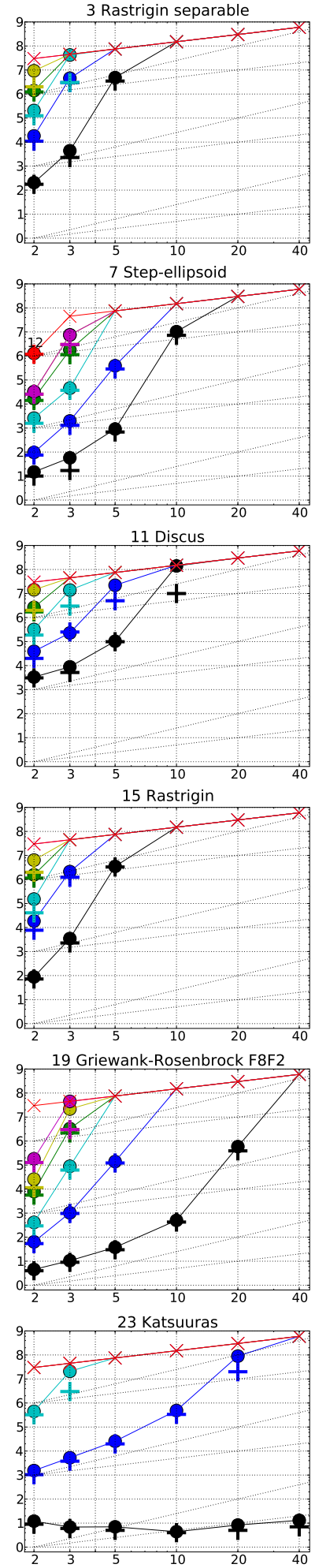
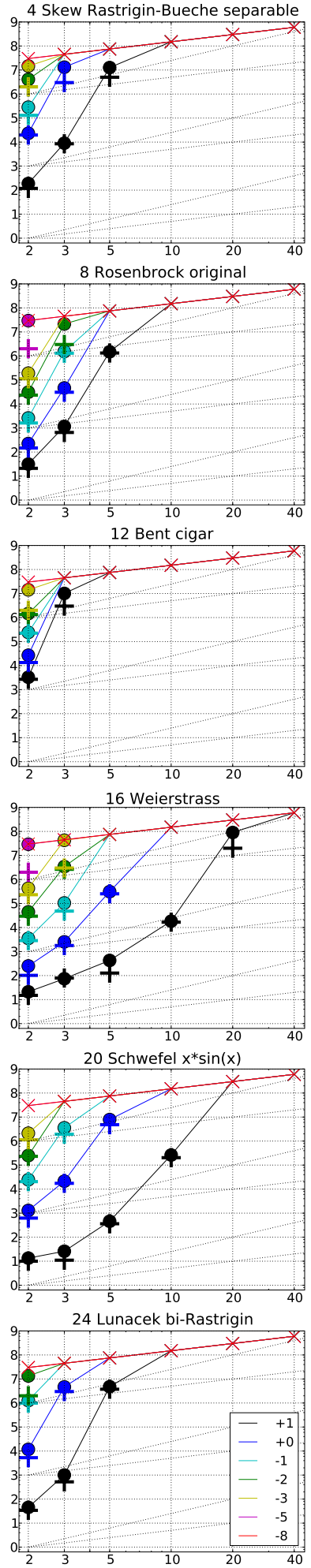

Figure 3: Expected Running Time (ERT, $\bullet$ ) to reach $f_{\mathrm{opt}}+\Delta f$ and median number of function evaluations of successful trials $(+)$, shown for $\Delta f=10,1,10^{-1}, 10^{-2}, 10^{-3}, 10^{-5}, 10^{-8}$ (the exponent is given in the legend of $f_{1}$ and $\left.f_{24}\right)$ versus dimension in log-log presentation. The $\operatorname{ERT}(\Delta f)$ equals to \#FEs $(\Delta f)$ divided by the number of successful trials, where a trial is successful if $f_{\mathrm{opt}}+\Delta f$ was surpassed during the trial. The \#FEs $(\Delta f)$ are the total number of function evaluations while $f_{\mathrm{opt}}+\Delta f$ was not surpassed during the trial from all respective trials (successful and unsuccessful), and $f_{\text {opt }}$ denotes the optimal function value. Crosses $(\times)$ indicate the total number of function evaluations \#FEs $(-\infty)$. Numbers above ERT-symbols indicate the number of successful trials. Annotated numbers on the ordinate are decimal logarithms. Additional grid lines show linear and quadratic scaling. 

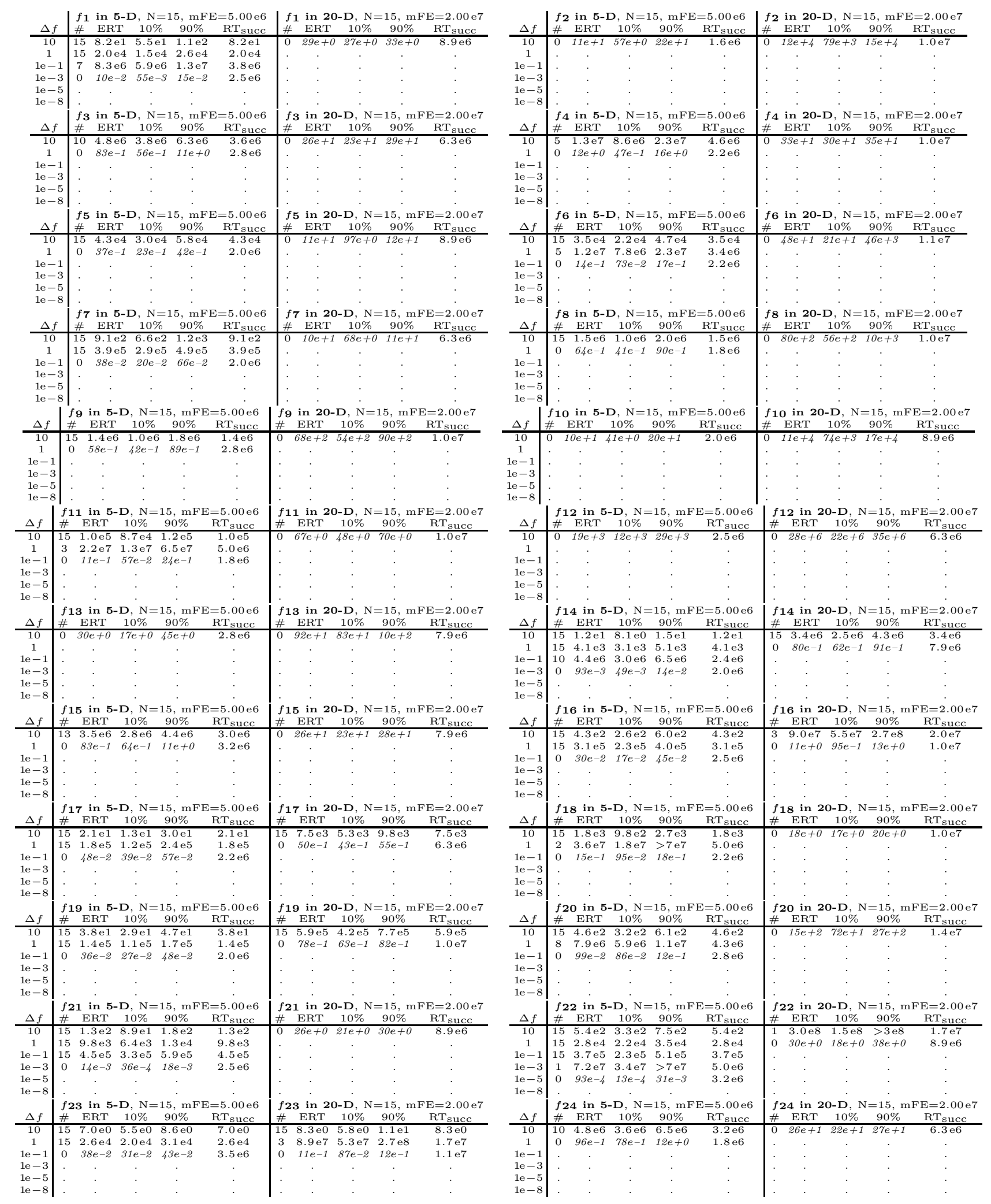

Table 1: Shown are, for a given target difference to the optimal function value $\Delta f$ : the number of successful trials $(\#)$; the expected running time to surpass $f_{\mathrm{opt}}+\Delta f$ (ERT, see Figure 3 ); the 10\%-tile and 90\%-tile of the bootstrap distribution of ERT; the average number of function evaluations in successful trials or, if none was successful, as last entry the median number of function evaluations to reach the best function value $\left(\mathrm{RT}_{\text {succ }}\right)$. If $f_{\mathrm{opt}}+\Delta f$ was never reached, figures in italics denote the best achieved $\Delta f$-value of the median trial and the $10 \%$ and $90 \%$-tile trial. Furthermore, $\mathrm{N}$ denotes the number of trials, and $\mathrm{mFE}$ denotes the maximum of number of function evaluations executed in one trial. See Figure 3 for the names of functions. 

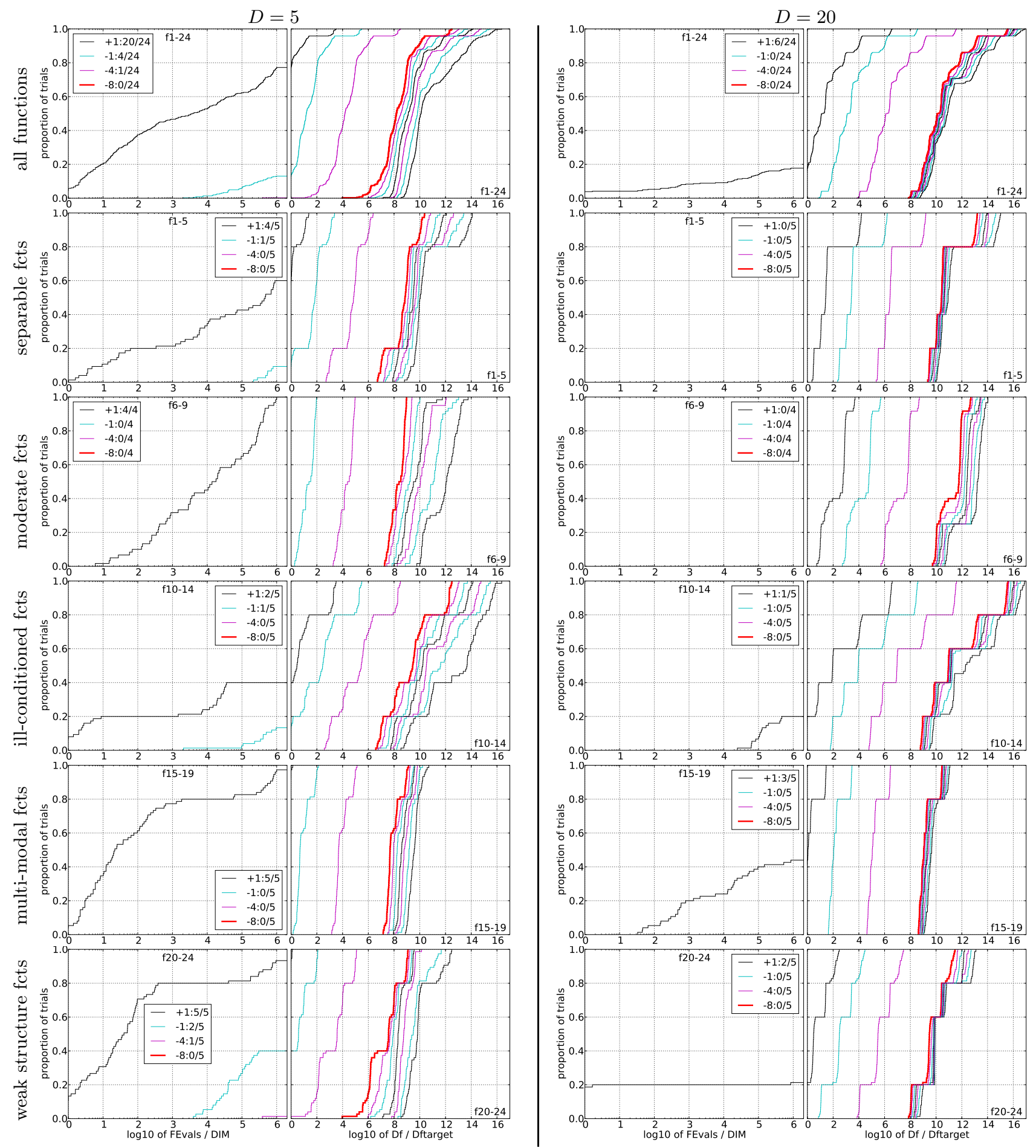

Figure 4: Empirical cumulative distribution functions (ECDFs), plotting the fraction of trials versus running time (left subplots) or versus $\Delta f$ (right subplots). The thick red line represents the best achieved results. Left subplots: ECDF of the running time (number of function evaluations), divided by search space dimension $D$, to fall below $f_{\mathrm{opt}}+\Delta f$ with $\Delta f=10^{k}$, where $k$ is the first value in the legend. Right subplots: ECDF of the best achieved $\Delta f$ divided by $10^{k}$ (upper left lines in continuation of the left subplot), and best achieved $\Delta f$ divided by $10^{-8}$ for running times of $D, 10 D, 100 D \ldots$ function evaluations (from right to left cycling black-cyan-magenta). Top row: all functions; second row: separable functions; third row: misc. moderate functions; fourth row: ill-conditioned functions; fifth row: multi-modal functions with adequate structure; last row: multi-modal functions with weak structure. The legends indicate the number of functions that were solved in at least one trial. FEvals denotes number of function evaluations, $D$ and DIM denote search space dimension, and $\Delta f$ and Df denote the difference to the optimal function value. 